\title{
A Tesla Transformer and a Coaxial Peaking Switch as a UWB Pulse Source
}

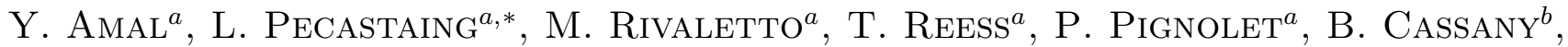 \\ A. Silvestre de Ferron $^{b}$, L. Courtois ${ }^{b}$ And E. Merle ${ }^{b}$ \\ ${ }^{a}$ Laboratoire de Génie Electrique, Université de Pau, 2 av. Angot, 64053 Pau, France \\ ${ }^{b}$ CEA CESTA, BP 2, Routes des Gargails, 33114 Le Barp, France
}

\begin{abstract}
This paper presents a high voltage pulse source which is able to generate ultra wideband (UWB) pulses during about $1 \mathrm{~ns}$ through a 16 antennas array. This UWB source is composed of a $50 \mathrm{kV}$ DC voltage supply, a Tesla transformer to amplify this voltage up to $400 \mathrm{kV}$, a gaseous pressurized peaking switch and an impedance transformer $(50 \Omega \rightarrow 3.125 \Omega$ ). This output impedance value corresponds to the input impedance value of a sixteen $50 \Omega$ antennas array. That is why a distributor is needed in order to feed the antenna array. In this paper, the peaking switch and the capacitive line divider used to characterise the generated pulses are particularly described. The peaking switch is based on the principle of a line discharge by means of a high pressure gas switch. It is loaded with a Tesla transformer to obtain a good pulse reproducibility. The main characteristics of the output pulse waveform (amplitude and rise time) are linked to the properties of the gas switch and particularly to the gap distance, the pressure and the nature of the gas used in the switch filling. The aim is to find a good compromise between various parameters as the output pulse amplitude, the rise time and the repetition rate in order to ensure a better efficiency of the UWB source. Classical voltage measurement techniques do not allow us an estimation of the main characteristics of such an output signal. Therefore a voltage probe was designed and realised to measure both the amplitude and the rise time of the pulses delivered by the generator. This device is based on the principle of a capacitive line divider. Calibration tests (transient and frequency tests) were performed and show that the high cut-off frequency, around $2.5 \mathrm{GHz}$, is consistent with the transient response of the output high voltage waveform. The design, realisation and calibration tests are also presented.
\end{abstract}

PACS numbers: $84.70 .+\mathrm{p}, 07.57 . \mathrm{Hm}, 84.30 . \mathrm{Ng}$

\section{Introduction}

The interest for high voltage closing switches on nanosecond time scales has risen in a range of applications including high power microwave, pulse laser or particle accelerators. Recent works have extended the feasibility of a broadband device for high electromagnetic field radiations. This kind of device consists of a high gain antenna driven by a self-contained, compact, repetitive and voltage adjustable generator. In this context, a prototype of a compact generator was developed. Its main characteristics are an output voltage in the range of $100-300 \mathrm{kV}$, not more than a few hundreds of picoseconds rise time and a repetition rate of a few tens $\mathrm{Hz}$.

\section{UWB pulse source description}

This UWB pulse source aims at generating impulses of a few hundreds $\mathrm{kV}$ with fast rise times from a DC continuous voltage of $50 \mathrm{kV}$. This source consists of the devices presented in Fig. 1:

- a $50 \mathrm{kV}$ DC voltage supply,

* corresponding author; e-mail: laurent.pecastaing@univ-pau.fr

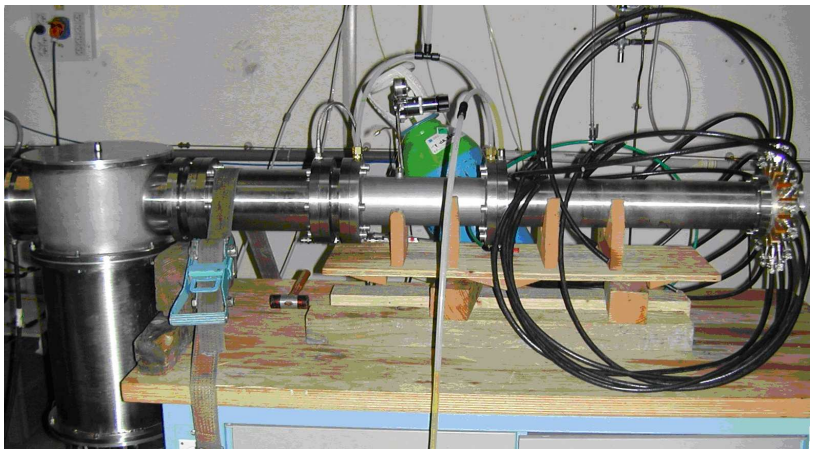

Fig. 1. Design of the UWB pulse source.

- a Tesla transformer to amplify this voltage and its driving system,

- a gaseous pressurized peaking switch,

- a capacitive line divider,

- an impedance transformer $(50 \Omega \rightarrow 3.125 \Omega)$,

- a distributor towards a sixteen $50 \Omega$ antennas array. 


\subsection{Design of the coaxial generator with its peaking stage}

The purpose of this component is to sharpen the rise time of the impulse generated by the air Tesla transformer in order to reach rise times close to $200 \mathrm{ps}[1,2]$. It consists of a spark-gap with pressurized gas but without air flow and a pressure being able to go up to 100 bars. The aim is to place this peaking switch on the curve of Paschen [3] where the conditions of the product $P$ (pressure $) \times d$ (gap distance) make it possible to reach breakdown voltage values of the peaking switch near the maximum of the output pulse generated by the air Tesla transformer. The gap distance can be adjusted from 0 to $5 \mathrm{~mm}$. The impedance of the input forming line is $30 \Omega$, whereas the output transmission line impedance is $50 \Omega$ to improve the pulse voltage amplitude available on the load.

\subsection{Design of the capacitive line divider}

The output voltage measurement is achieved by probes made of a capacitive line divider [3] associated with attenuators.

A complete calibration (transient and frequency responses) test was performed on the divider used in this experiment. The transient response to an ultra short pulse is very important. Our vector network analyser (VNA Anritsu MS4623B) makes it possible to simulate the transient response of an impulse close to a Gaussian pulse. The VNA simulates pulses with a rise time of $97 \mathrm{ps}$ and duration at half width of $180 \mathrm{ps}$. The reference pulse measurement and the measure with capacitive divider give very close characteristics: the rise time (10\% to $90 \%$ ) is now 104 ps and the width at half maximum is 205 ps. However this time-domain calibration is not sufficient to determine the characteristics of this probe accurately. A frequency calibration is also needed to complete this first result.

The VNA measures the frequency response of the probes to complete the first calibration test. The transmission and reflection parameters were studied up to $3 \mathrm{GHz}$. The transmission parameter obtained is linear and constant in regard to the frequency. The transmission coefficient of the probe used in our experiments is about $-62 \mathrm{~dB}$ up to $3 \mathrm{GHz}$. The measurement of this parameter is presented in Fig. 2. It is compared with the results issued from the transient analysis. Besides, the reflection parameter is always lower than $-14 \mathrm{~dB}$.

The main characteristics of the divider for our application are an upper bandwidth of $2.9 \mathrm{GHz}$ and an attenuation of $-62 \mathrm{~dB}$. It enables pulse measurement with a rise time as short as 130 ps. This value is shorter than the fastest rise time measured in this study.

\subsection{Design of the impedance transformer}

The purpose of this element is to gradually transform the characteristic impedance [5]. In our case, the aim consists of passing from an input impedance of $50 \Omega$ to

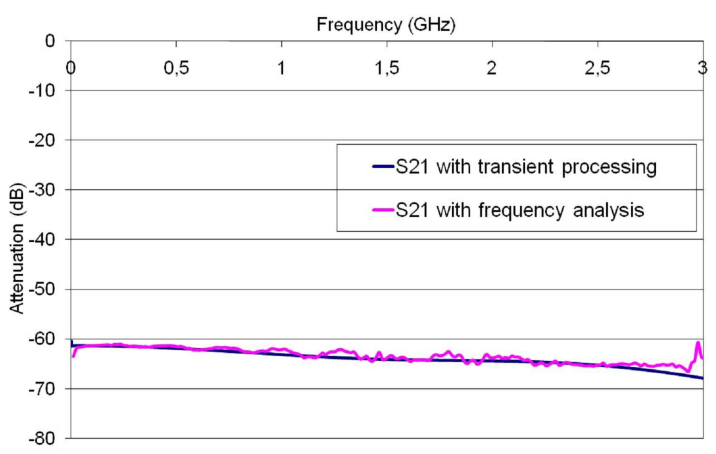

Fig. 2. Comparison between the transmission coefficient and the results obtained from the transient analysis.

an output impedance of $3.125 \Omega$, corresponding to the impedance presented by 16 cables in parallel connected to a 16 antennas array.

\section{Experimental results}

Figure 3 shows the typical waveform of the charging voltage of the peaking stage driven by the transformer. The combination of the Tesla transformer and the peaking stage delivers a monopolar impulse. For a better efficiency, the breakdown of the peaking spark gap must occur during the second alternation. Whereas this condition can be assured with a low pulse repetition frequency $(\mathrm{PRF})$, it becomes more delicate when the PRF is increased up to $200 \mathrm{~Hz}$.

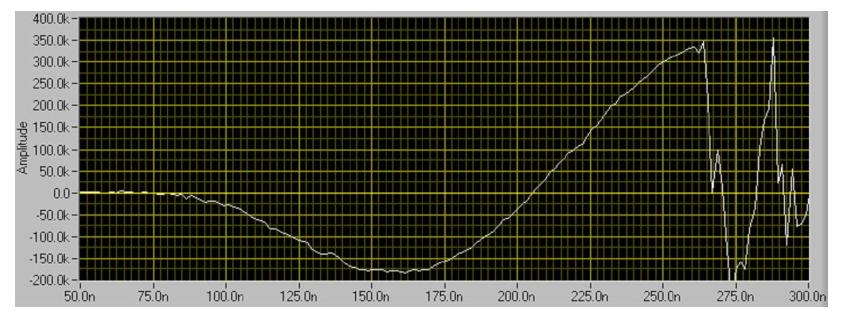

Fig. 3. Typical waveform of the transformer output voltage to drive the peaking switch.

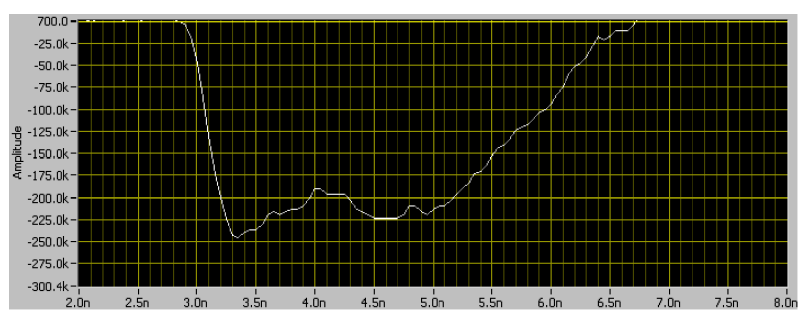

Fig. 4. Typical waveform after the peaking switch (rise time $=250 \mathrm{ps}, V_{\text {OUT }}=240 \mathrm{kV}$ ).

A typical output pulse is presented in Fig. 4. The rise time is approximately $250 \mathrm{ps}$ and the pulse width at 
half maximum of 2.5 ns. The pulse amplitude is close to $250 \mathrm{kV}$.

\section{Conclusions}

Our ultra wideband pulse source combines a Tesla transformer and a monopolar peaking stage based on a pressurised gaseous switch. The tests are carried out very satisfactorily to obtain an output voltage in the range of $100-300 \mathrm{kV}$, not more than 250 ps of rise time and a repetition rate of a few tens $\mathrm{Hz}$. However, to take into account a criterion of reliability, the pulse source will have to undergo complementary experiments for a technological validation.

\section{References}

[1] G.A. Dawson, D.E. Davies, Research Notes, Brit. J. Appl. Phys. 14, 155 (1963).

[2] E.E. Kunhardt, W.W. Byszewski, Phys. Rev. A 21, 2069 (1980).

[3] L. Pécastaing, J. Paillol, T. Reess, A. Gibert, P. Domens, IEEE Trans. Plasma Sci. 34, 1822 (2006).

[4] L. Pécastaing, J. Paillol, T. Reess, A. Gibert, P. Domens, Measurement Sci. Techn. 12, 1718 (2001).

[5] V.I. Koshelev, V.P. Gubanov, A.M. Efremov, S.D. Korovin, B.M. Kovalshuk, V.V. Plisko, A.S. Stepchenko, K.N. Sukhushin, Proc. 2nd Intern. Congress on Radiation Physics, High Current Electronics and Modification of Materials, Tomsk, 258 (2006). 